\title{
Evolution of the Polymorph Selectivity of Titania Formation under Acidic and Low-Temperature Conditions
}

\author{
Gunnar Símonarson, ${ }^{\dagger}$ Sanna Sommer, ${ }^{\ddagger}$ Antiope Lotsari, ${ }^{\dagger}$ Björn Elgh, ${ }^{\dagger}$ Bo B. Iversen, ${ }^{\ddagger}$ \\ and Anders E.C. Palmquist* ${ }^{*}+(1)$ \\ ${ }^{\dagger}$ Applied Chemistry, Department of Chemistry and Chemical Engineering, Chalmers University of Technology, 41296 Göteborg, \\ Sweden \\ ${ }^{\ddagger}$ Center for Materials Crystallography, Department of Chemistry and iNANO, Aarhus University, Langelandsgade 140, 8000 Aarhus, \\ Denmark
}

\section{Supporting Information}

ABSTRACT: Evolution of the polymorph selectivity of titanium dioxide was studied under acidic and low-temperature synthesis conditions. Short synthesis times resulted in high relative amounts of the rutile phase, and long synthesis times resulted in high relative amounts of the brookite and anatase phases. The effect of titania precursor concentration was investigated and found to have a large impact on the polymorph selectivity. As the reaction proceeds with time, changes in the chemical environment, caused in particular by the gradually decreasing titania precursor concentration, are therefore likely the cause of the change in polymorph selectivity observed.

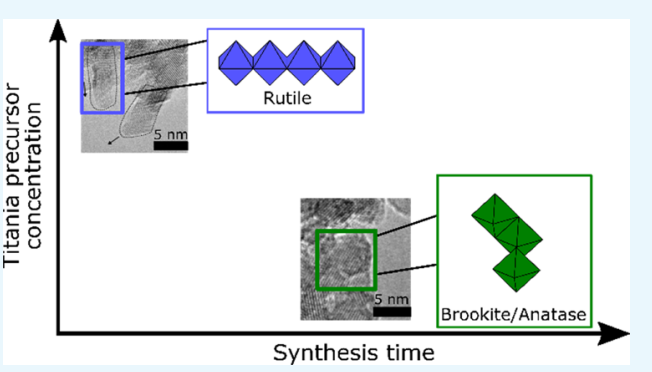

\section{INTRODUCTION}

Nanostructured titanium dioxide or titania $\left(\mathrm{TiO}_{2}\right)$ has received much attention from industry as well as the research community during the past decades as a functional material for numerous applications including sustainable energy production, ${ }^{1,2}$ self-cleaning surfaces, ${ }^{3}$ osseointegration support, ${ }^{4}$ and catalysis. ${ }^{5}$ This continuous interest in nanostructured titania and titania-containing nanocomposites has spurred the development of methods to synthesize them under versatile conditions, for example, at low temperature, under different $\mathrm{pH}$, and from different precursors. As a consequence, nowadays, a broad range of synthesis routes to nanostructured titania is available including sol-gel routes, ${ }^{6,7}$ hydrothermal routes, ${ }^{8-10}$ and micelle and inverse micelle routes. ${ }^{11,12}$ On the basis of the microemulsion system, a low-temperature synthesis of crystalline mesoporous titania with good polymorph control has been previously developed. ${ }^{13,14}$ In these studies, it was shown that $\mathrm{pH}$, temperature, and synthesis time influenced the polymorph distribution. ${ }^{13-15}$

Controlling the crystallinity and the polymorph distribution of nanostructured titania is of paramount importance for application-targeted material design. Anatase, rutile, and brookite are the three main crystal polymorphs of titania of which rutile is the thermodynamically most stable polymorph in bulk titania. ${ }^{16-18}$ However, low-temperature wet chemical synthesis routes frequently produce titania in the thermodynamically less favored anatase phase. In general, the synthesis of thermodynamically less stable phases is achieved by kinetically controlled synthesis routes. It has, however, been predicted that anatase and brookite can be thermodynamically favored over rutile when crystallite sizes are of a few nanometers in size. ${ }^{19}$ Banfield et al. suggested that the formation of nanosized titania crystallites in the anatase and brookite phases could be explained by a reversal of the thermodynamic stability for titania crystallites smaller than 35 $\mathrm{nm} .^{20}$ They predicted that brookite was the thermodynamically most stable phase between 11 and $35 \mathrm{~nm}$ and anatase below 11 $\mathrm{nm}{ }^{16,20}$ Their predictions were at a sufficiently small crystallite size, the high-surface free energy of rutile compared to anatase becomes dominant over the difference in bulk free energy and thus reverses the relative thermodynamic stability of anatase and rutile. The surface free energy of brookite was estimated to be in a similar range as the other two phases. It should be noted that these predictions do not take into account the effect of the chemical environment of the nanoparticles, which may have a significant contribution to the relative thermodynamic stabilities of the phases. To our knowledge, the reversed thermodynamic stability of the titania phases has not hitherto been experimentally verified.

Here, we report a systematic study of a low-temperature low$\mathrm{pH}$ synthesis of titania and experimentally demonstrate an apparently thermodynamically driven formation of brookite and anatase nanocrystals. This opens up a new avenue of synthesis procedures for polymorph-selective synthesis of nanocrystalline titania materials. We investigate the influence of variables, such as the synthesis time and titania precursor concentration, and assess the effects of time-evolving changes in chemical composition of the synthesis mixture resulting from the progression of the reaction.

Received: December 7, 2018

Accepted: February 12, 2019

Published: March 25, 2019 
Table 1. Compositions and Reaction Times of the Synthesis Mixtures Used To Prepare Titania Nanoparticles

\begin{tabular}{|c|c|c|c|c|c|c|c|c|c|}
\hline synthesis time (days) & 3 & 5 & 7 & 11 & 14 & 24 & 32 & 39 & 56 \\
\hline$[\mathrm{Ti}]=0.65 \mathrm{M}$ & A1 & $\mathrm{A} 2$ & $\mathrm{~A} 3$ & A4 & A5 & A6 & A7 & A8 & A9 \\
\hline$[\mathrm{Ti}]=0.33 \mathrm{M}$ & & & $\mathrm{B} 1$ & & B2 & & B3 & & B4 \\
\hline$[\mathrm{Ti}]=1.12 \mathrm{M}$ & & & $\mathrm{C} 1$ & & $\mathrm{C} 2$ & & $\mathrm{C} 3$ & & $\mathrm{C} 4$ \\
\hline
\end{tabular}

\section{RESULTS AND DISCUSSION}

Three series of titania samples were prepared according to the procedures described in the Experimental Methods section and summarized in Table 1.

Diffractograms of samples A1 to A9 are shown in Figure 1a. The Bragg peaks that were mainly used to identify the distinct

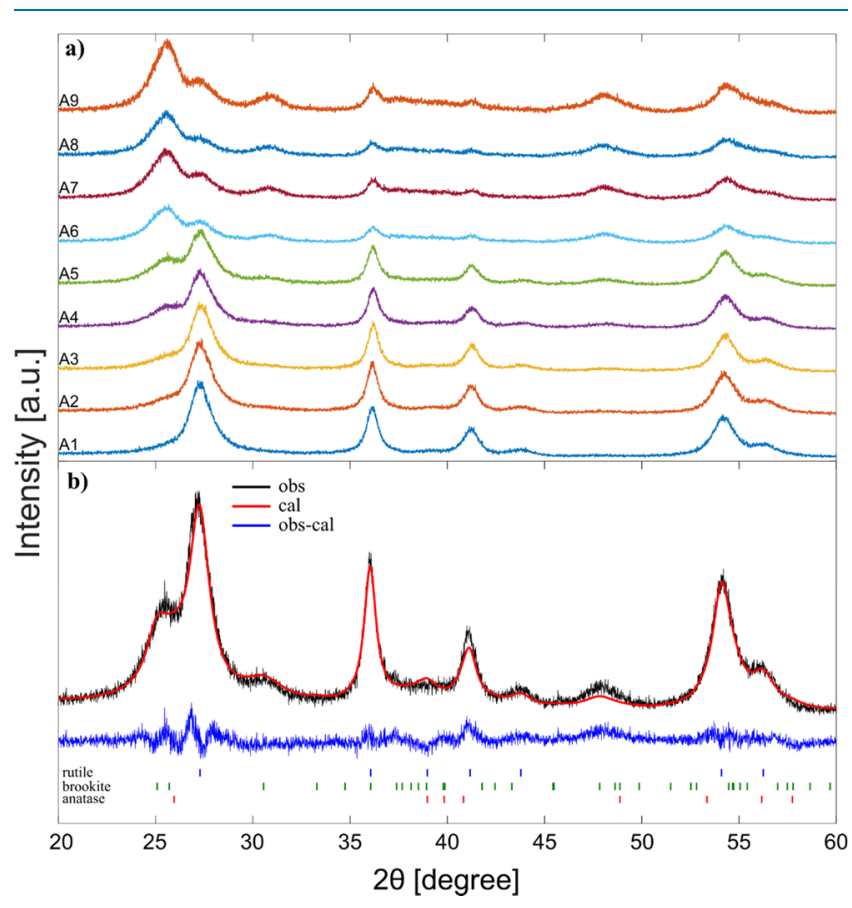

Figure 1. (a) X-ray diffractograms of samples A1-A9. The diffractograms are in the order of increasing reaction time, from bottom to top, and offset in intensity for clarity. (b) Rietveld refinement of sample A5. The observed pattern is shown in black, calculated pattern in red, and the difference between observed and calculated is shown below in blue. The Bragg peak positions for each phase are indicated below.

phases of titania are observed at $2 \theta=27.4^{\circ}$ (rutile $(110)$ ), and a set of peaks that originate from three reflections, one from anatase (101) and two brookite reflections (120) and (111) are observed at approximately $2 \theta=25.3^{\circ}$. Additionally, the Bragg peak at $2 \theta=30.8^{\circ}$ corresponding to the brookite (121) reflection is observed for samples A5-A9. The diffractograms clearly display a shift from mainly rutile phase for sample A1 toward a progressively higher content of titania in the anatase and brookite phases for samples A4-A9. Interestingly and contrary to the normally observed increase in selectivity for rutile formation at prolonged reaction times, ${ }^{14,24}$ we here observe a progressive increase of anatase and brookite accompanied by a relative decrease of the rutile phase. However, this apparently agrees with the predictions by Banfield et al. of the existence of a thermodynamic driving force for the formation of anatase and brookite for crystallites below $11 \mathrm{~nm}$ and between 11 and $35 \mathrm{~nm}$, respectively. ${ }^{20}$ Because of the peak overlap of the anatase and brookite phases, the phase identification by simple peak indexing can be difficult, making modeling of the PXRD essential. However, the peak at $30.8^{\circ}$ confirms that brookite is present in samples A5-A9. To properly identify crystalline phases and to quantify the phase composition in each sample, the Rietveld refinements were performed. Figure $1 \mathrm{~b}$ shows the refinement of sample A5, where the observed (obs) and calculated (cal) patterns are presented as well as the difference between the two (obs-cal). The Bragg peaks of each phase are shown below the diffractograms. The morphology of the crystals was also obtained and is presented in the Supporting Information (Figure S1). The refined parameters, including crystal sizes, spherical harmonic coefficients, and the unit cell parameters, are reported in the Supporting Information.

Figure 2 shows quantitatively how the weight fraction of each crystal phase develops with synthesis time for series A from 3 to 56 days. It is worth pointing out that after 39 days, the reaction yield is measured to be $95 \%$, but due to possible material loss in the washing process, which is not accounted, the conversion of the titania precursor is likely closer to $100 \%$. Because almost all the precursor has been transformed to titania particles at that point, changes in phase composition are unlikely hereafter in this low-temperature synthesis.

The fractions of the phases are obtained from the Rietveld refinements of the X-ray diffractograms of each sample. In Figure 2, it can be observed that the relative weight fraction of rutile decreases quite rapidly from 88 to $26 \%$ between 3 and 24 days of synthesis time, after which the decrease is slower and finally the relative fraction stabilizes at $13 \%$ after 39 days. Conversely, the relative weight fraction of brookite and anatase is initially low but increases with increasing synthesis time. The fraction of brookite increases from 3 to $62 \%$ between 3 and 39 days of synthesis time, and the fraction of anatase stabilizes at $25 \%$ after 39 days. This time-dependent polymorph selectivity indicates that rutile is initially formed by a kinetically driven mechanism, which is dominant in the earlier stage of the reaction, whereas anatase and brookite phases are formed at later stages in what may appear to be thermodynamically favored mechanisms. Although it can be observed from Figure 2 that the phase composition changes with synthesis time, it is not clear if the rutile phase is transformed to anatase and brookite, or if they are formed separately from the unreacted precursor. It should also be noted that the fractions shown here are relative weight fractions of the three crystalline phases and do not contain information about the amorphous phase.

To determine the absolute amount formed of each crystal phase at any given time of the synthesis, the conversion yield of the reaction has to be taken into account. In addition to that, to account for the amorphous phase, additional XRD measurements with a quantitative internal standard were conducted. The nature of the amorphous phase has not been studied in detail in the present work, but other studies have reported that apparently amorphous structures may have an atomically ordered core and a large number of defects closer to the surface of the particle. In these studies, in situ total X-ray scattering measurements ${ }^{10,25}$ and wide-angle X-ray scattering 


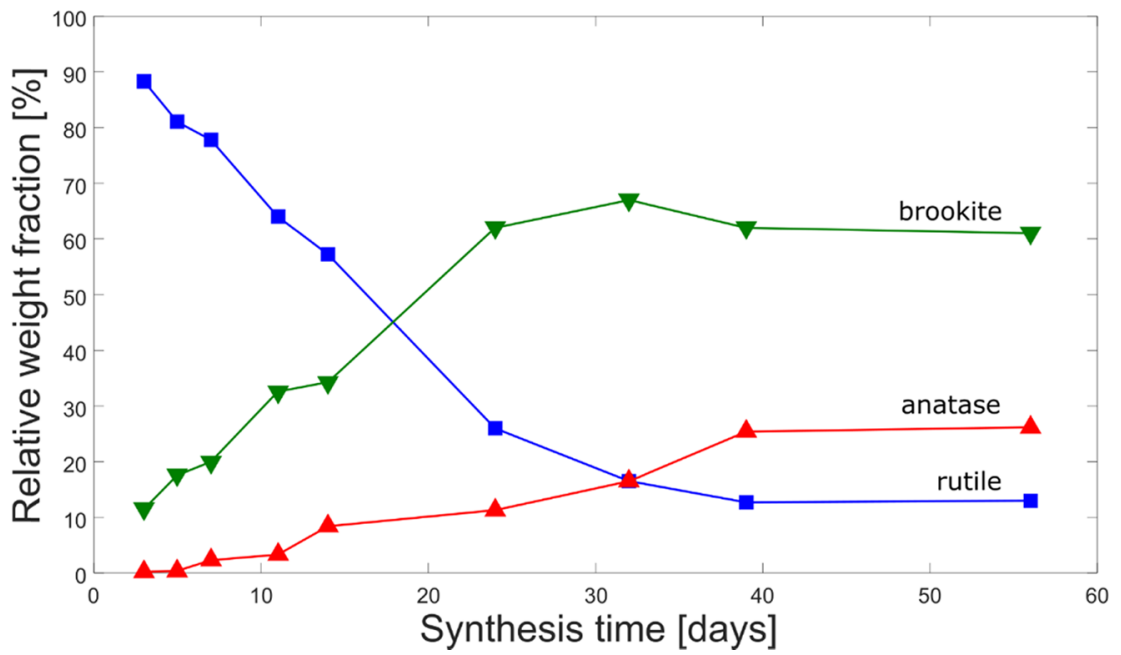

Figure 2. Relative weight fraction of rutile (blue squares), brookite (green down-pointing triangles), and anatase (red up-pointing triangles) in series A. The weight fractions were extracted from the Rietveld refinements of the diffractogram of each sample.

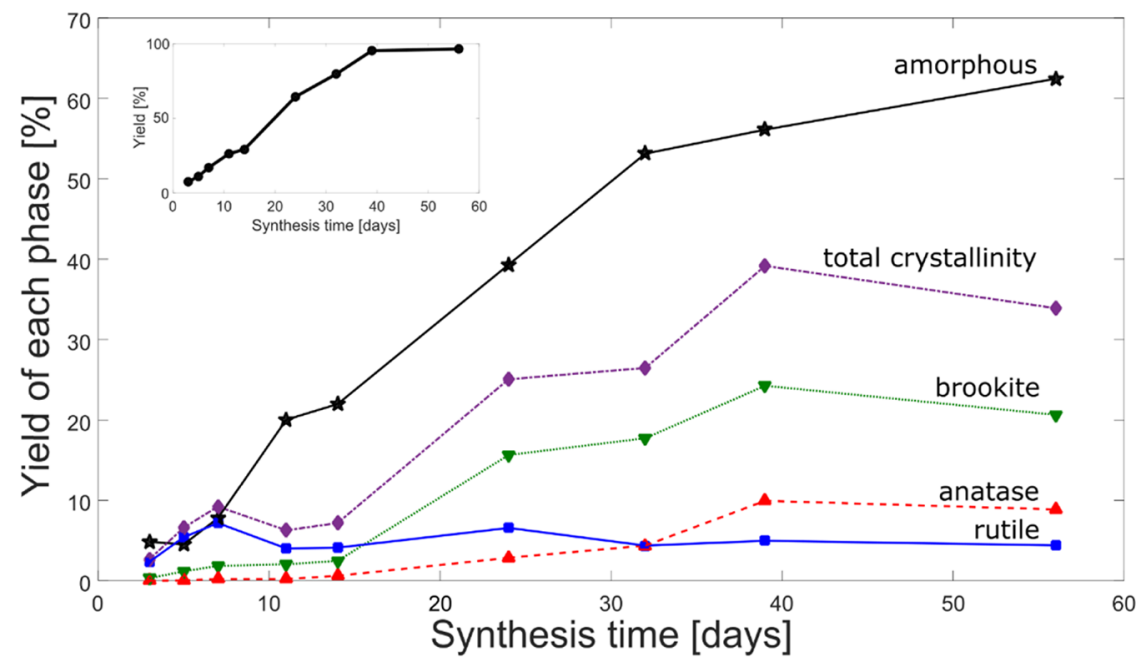

Figure 3. Time evolution of the phase formation in series A. The yield of the phases is calculated by multiplying the relative concentration of each phase with the total yield of the reaction. The total crystallinity (purple diamonds) is the sum of the rutile (blue squares), anatase (red up-pointing triangles), and brookite (green down-pointing triangles) phases. The yield of the amorphous phase (black stars) increases during the whole synthesis time. The inset shows the total yield of the reaction.

with PDF analysis ${ }^{26}$ were used to describe the amorphous phase. The latter study included the formation of amorphous titania nanoparticles, which were described as having a strained anatase-like core with a highly distorted shell. The average coordination number of titanium was estimated lower and the $\mathrm{Ti}-\mathrm{O}$ bond length shorter than in bulk titania, which was attributed to features in the distorted shell.

Figure 3 shows the yield of each phase where on the $y$-axis, the weight fraction is multiplied with the total yield of the reaction. The total yield was measured by dividing the mass of the retrieved titania particles, after centrifuging, washing, and drying, with the theoretical mass as if $100 \%$ conversion of the titania precursor was achieved. The inset shows the total yield of the reaction, which approaches $100 \%$ after 39 days. It can be seen that of the three crystal structures, mainly rutile is initially formed, and after 11 days, the absolute amount of rutile remains nearly constant. From 14 days, the anatase and brookite polymorphs are mainly formed. This indicates that the rutile crystals are not transformed to the other crystal structures, but rather that the crystallization of anatase and brookite occurs separately at the later stages of the reaction. The amorphous phase is formed continuously throughout the whole synthesis time and is the predominant phase from 11 days onward.

To rationalize the drastic change in polymorph selectivity observed in Figures 1 and 2, we need to understand what factors may influence this. From previous studies, it is known that several factors, such as the type of titania precursor and its concentration, $\mathrm{pH}$, type of acid, and temperature, impact the crystal structure formation for $\mathrm{TiO}_{2}{ }^{8,14,15,27}$ In our system, the concentration of unreacted titania precursor decreases as the reaction proceeds and titania particles form and there is also a change in $\mathrm{pH}$ as the precursor is first hydrolyzed, and then as a result of condensation reactions between molecular titania precursors and the surface of the growing particles. More specifically, we note that the initial precursor concentration in series $\mathrm{A}$ is $0.65 \mathrm{M}$ and at this stage, mainly rutile forms. Because of particle formation, the concentration of molecular titania precursor species decreases to $0.45 \mathrm{M}$ after 14 days, and from this point forward, mainly brookite and anatase crystals 

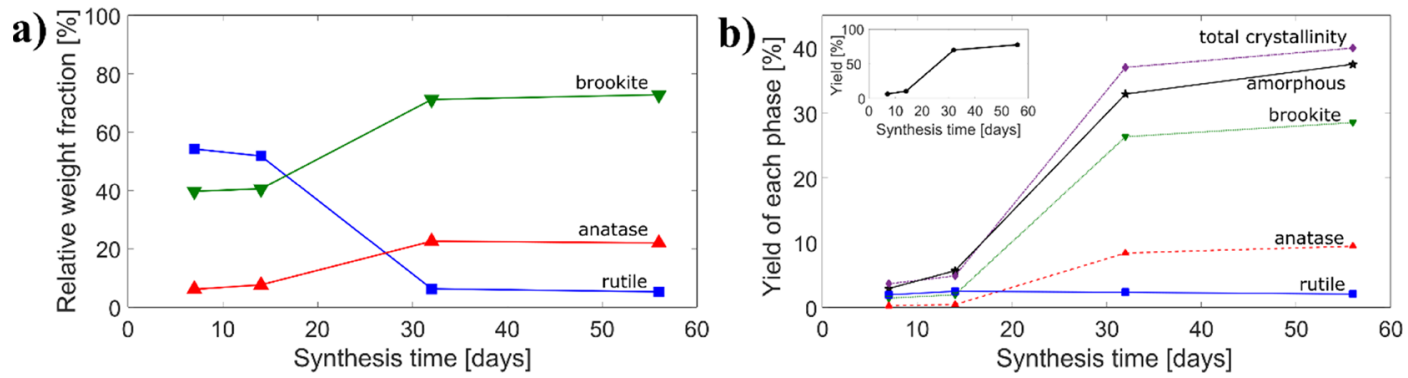

Figure 4. Time evolution of the phase formation in series B. (a) Relative weight fraction of rutile (blue squares), brookite (green down-pointing triangles), and anatase (red up-pointing triangles). The weight fractions were extracted from the Rietveld refinements of the diffractogram of each sample. (b) The total crystallinity (purple diamonds) is the sum of the rutile (blue squares), anatase (red up-pointing triangles), and brookite (green down-pointing triangles) phases. The yield of the amorphous phase (black stars) increases during the whole synthesis time. The inset shows the total yield of the reaction.
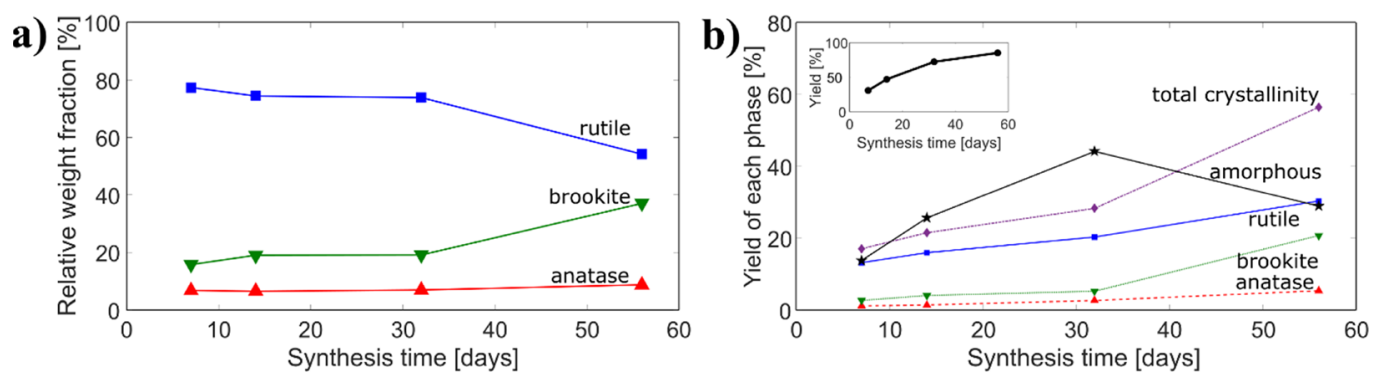

Figure 5. Time evolution of the phase formation in series C. (a) Relative weight fraction of rutile (blue squares), brookite (green down-pointing triangles), and anatase (red up-pointing triangles) in series C. The weight fractions were extracted from the Rietveld refinements of the diffractogram of each sample. (b) The total crystallinity (purple diamonds) is the sum of the rutile (blue squares), anatase (red up-pointing triangles), and brookite (green down-pointing triangles) phases. The yield of the amorphous phase (black stars) is lower than the total crystallinity at 56 days. The inset shows the total yield of the reaction.

form. To investigate our hypothesis that the concentration of molecular titania precursor species influences the polymorph selectivity, two additional series of samples were prepared. In series $\mathrm{B}$ and $\mathrm{C}$, the initial precursor concentration was lower and higher, respectively, compared to series A. The effect of the initial titania precursor concentration on the polymorph selectivity is illustrated in Figures 4 and 5.

Figure $4 \mathrm{a}$ shows how the relative weight fraction of each crystal phase changes with synthesis time in series $B$, where the initial titania precursor concentration is $0.33 \mathrm{M}$. A synthesis time of $7-14$ days results in $50-55 \%$ rutile relative weight fraction, whereas for synthesis time of 32-56 days, it decreases to about $5 \%$. The brookite fraction is $40 \%$ for a synthesis time of 7-14 days after which it increases to $70 \%$ for $32-56$ days. Similarly, the anatase fraction increases from $5 \%$ to about $22 \%$ from synthesis time of 7 to 56 days. This is a similar trend as seen in series A, where the fraction of rutile decreases with increasing synthesis time, and at the same time, the fraction of anatase and brookite increases. However, in series B, the fraction of rutile is at all points much lower than in series $A$.

Figure $4 \mathrm{~b}$ shows the yield of each phase in series $\mathrm{B}$. The inset shows the total yield of the reaction, which is only about $10 \%$ after 14 days but reaches $85 \%$ after 56 days. It means that although the relative weight fraction of rutile during the first 14 days is quite high, the absolute amount of rutile formed is very low, where the yield of the phase is less than 3\% after 56 days. After 14 days, mainly brookite and anatase crystal phases are formed along with the amorphous phase. With the initial lower titania precursor concentration $[\mathrm{Ti}]=0.33 \mathrm{M}$, series $\mathrm{B}$ yields much less rutile than series A. Interestingly, the yield of total crystallinity in this series is slightly higher than that in series A, and after 56 days, the sample contains more than $50 \%$ crystalline material.

Figure 5a shows how the relative amount of each crystal phase changes with synthesis time in series $C$, where the initial titania precursor concentration is $1.12 \mathrm{M}$. The relative weight fraction of rutile is $77 \%$ after 7 days and decreases to $55 \%$ after 56 days. Between 7 and 56 days, the concentration of brookite increases from 15 to $37 \%$, but the increase in anatase concentration is much lower from 7 to $9 \%$. Although the trend of decreasing fraction of rutile with increasing synthesis time is observed, similar as for series $\mathrm{A}$ and $\mathrm{B}$, the rutile fraction is much higher in series $C$ than those of the other two series throughout the synthesis time.

Figure $5 \mathrm{~b}$ shows the yield of each phase in series $\mathrm{C}$. The inset figure shows the total yield of the reaction, which is $85 \%$ after 56 days. In the graph, it can be seen that of the three crystal phases, mainly rutile has formed after 7 day synthesis time, and it continues to form during the whole synthesis time. Lesser amounts of anatase and brookite are formed until 32 days, but between day 32 and 56, more brookite is formed. Here, the amorphous phase is formed until 32 days after which it decreases significantly, and its yield is half of the total crystallinity after 56 days. This decrease in the amorphous phase after 32 days, accompanied with a quite rapid increase in a brookite yield, indicates that it may be converted mainly to the brookite phase.

The results from series $\mathrm{B}$ and $\mathrm{C}$ confirm our hypothesis that the concentration of molecular titania precursor species influences the polymorph selectivity, where high concentration favors rutile and low concentration favors brookite and anatase. To explain the driving force for the polymorph selectivity in 
the three series, we need to understand how the chemical composition of the reaction mixtures changes as the reaction proceeds. The most obvious change is that as solid titania particles are formed, the concentration of the dissolved molecular titania precursor species in the solution decreases. To illustrate the effect that this has on the selectivity, the relative accumulated weight fraction of phases formed from the start of the syntheses is presented in Figure 6 as a function of

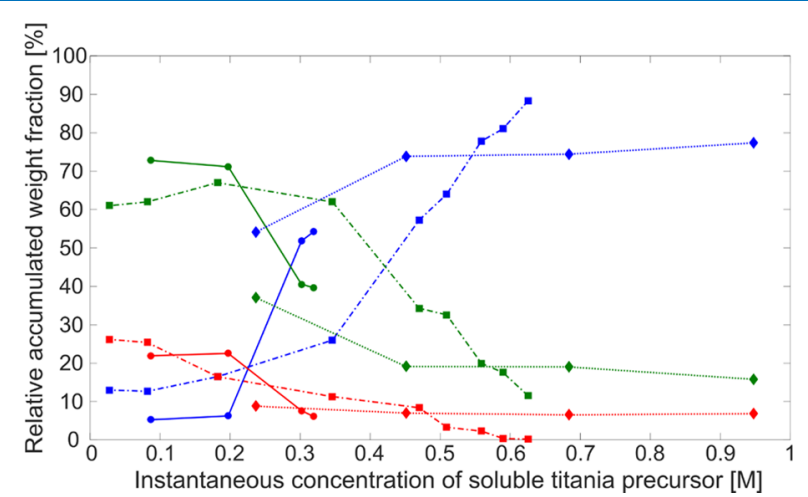

Figure 6. Relative accumulated weight fraction of rutile (blue), brookite (green), and anatase (red) in series A (squares), series B (circles), and series C (diamonds) formed from the start of the syntheses as a function of the instantaneous concentration of soluble titania precursor.

the instantaneous concentration of soluble titania precursor for all three series. The instantaneous concentration of soluble titania precursor in the solution at the sampling points is calculated by subtracting the amount of titania precursor that has been converted to solid phases from the initial titania precursor used in each series. The instantaneous concentration of soluble titania precursor used in the graph is the average between two subsequent sampling points. Differentiating the total crystallinity into the three crystalline polymorphs reveals that the selectivity of their formation strongly depends on the instantaneous titania precursor concentration in the solution. From the data, it is obvious that under the synthesis conditions employed here, the selectivity for rutile formation is highest when the titania precursor concentration is high. As the instantaneous concentration of the precursor decreases, the selectivity changes in favor of brookite at intermediate and low concentrations. The selectivity for anatase is low throughout the synthesis but increases somewhat at low precursor concentrations. In all three series, it was observed that the material formed is partly amorphous. From the data, we cannot, however, unambiguously distinguish clear trends of the relative fraction of amorphous versus the total crystalline material with instantaneous precursor concentration.

As a further attempt to illustrate the selectivity dependence on the precursor concentration, the formation rate of the polymorphs is plotted versus the instantaneous concentration of soluble titania precursor in Figure S4 of the Supporting Information. Although the data is scattered due to uncertainty in quantifying the amorphous phase and the relatively small changes in phase composition between sampling points, it confirms the selectivity dependence discussed above.

It should be noted that the proton concentration in the solution also changes as the reaction proceeds. Initially, when the titanium butoxide is added to the acidic solution, it is rapidly hydrolyzed, and due to excess water in the reaction mixture, the precursor is expected to be fully hydrolyzed. ${ }^{28}$ Under the acidic conditions in the system studied here, where hydroxo-aquo precursor species are present, the condensation reaction will proceed mainly via olation, leading to formation of hydroxo bridges (Ti-OH-Ti). ${ }^{29}$ As the condensation reactions proceed and titanium dioxide particles form, the oxygen bridge will not carry a proton in the final structure, and the proton will be released to the solution, decreasing its $\mathrm{pH}$. This was confirmed by monitoring the $\mathrm{pH}$ of the solution, which decreased with increasing synthesis time in all three series. The parallel change in proton and soluble titania precursor concentrations complicates the interpretation of the polymorph distribution and selectivity data. It has previously
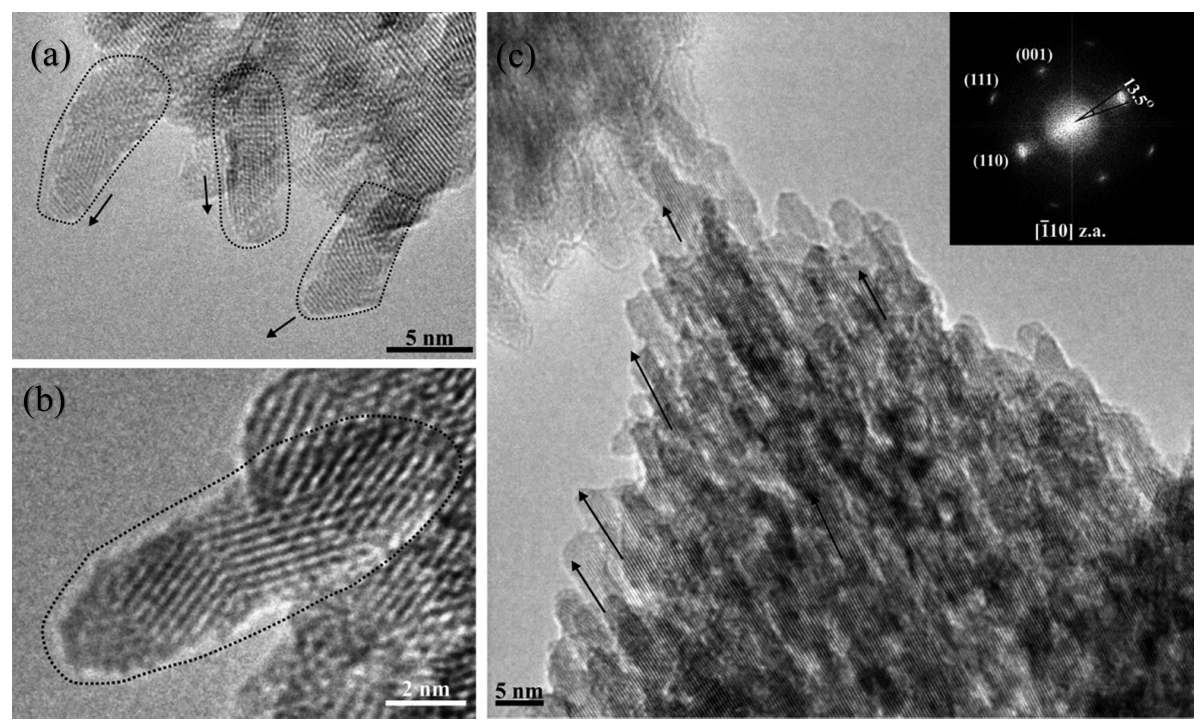

Figure 7. (a, b) HRTEM images of individual crystals and (c) a lower magnification HRTEM image of sample A1 (3 day synthesis time). In (a), the arrows denote the [001] direction aligned with the crystal long axes. (c) Image shows that the sample consists of needles that are roughly along the $c$-axis (denoted by the black arrows), and the inset is the FFT diffractogram showing that the majority of the crystals are oriented along the $[\overline{1} 10]$ zone axis. 
been shown, however, that under similar conditions as used here, a lower $\mathrm{pH}$ favors formation of rutile. ${ }^{14,15} \mathrm{We}$, therefore, conclude that the shift in selectivity to brookite and anatase toward the end of the syntheses found in Figures 2, 4, and 5 is not due to the decrease in $\mathrm{pH}$. Instead, the selectivity change appears due to the decrease in instantaneous concentration of soluble titania precursor according to Figure 6. The observation of an initial selectivity for rutile phase formation and a later preference for brookite and anatase phases is contradictory to the more commonly observed scenario where rutile forms with slower crystallization rates and where rapid crystallization favors anatase formation, usually attributed to kinetic formation of anatase crystals and thermodynamic formation of the rutile crystal structure. ${ }^{24}$

To further understand the mechanism of formation of the different polymorphs in the present system, we extracted the crystallite sizes from the Rietveld refinements and found that they were below $11 \mathrm{~nm}$. More specifically, the size of the rutile crystallites was estimated to be $5.6-7.2 \mathrm{~nm}$, and the crystals appear not to grow further in size during the synthesis time where the brookite crystallites were $2.5-5 \mathrm{~nm}$ and the anatase crystallites were $2.5-5.6 \mathrm{~nm}$ with a small variation in size with synthesis time. Below $11 \mathrm{~nm}$ in size, anatase and brookite have been predicted to be more thermodynamically stable than rutile. ${ }^{16,20}$ If the polymorph formation was thermodynamically controlled, it would be expected that mainly brookite and anatase would crystallize in this size range. To characterize the morphology and further evaluate the crystalline phases present in the samples, HRTEM imaging and SAED were used. Figure 7 shows HRTEM images of sample A1 (3 day synthesis time). The images clearly show lattice fringes, and images in Figure $7 \mathrm{a}, \mathrm{b}$ show that the size of the needle-shaped crystals is about $10 \mathrm{~nm}$. Figure 7c shows a bundle of crystals that are superimposed on each other, and the elongated crystals are roughly aligned along their long axis. The black arrows denote the direction that they are aligned, and from the fast Fourier transform (FFT) diffractogram (inset), it can be seen that the alignment of the crystals is along their $c$-axis. The arc-shaped (110) reflection corresponds to a misorientation along the [110] direction that was measured to $13.5^{\circ}$. Indexing the FFT confirms the $\mathrm{TiO}_{2}$ phase as rutile, in agreement with XRD analysis.

Figure 8 shows TEM images and SAED patterns of sample A9 (56 day synthesis time). The average size of the crystals is $4.5 \pm 1.5 \mathrm{~nm}$. The sample appears to be a combination of crystals of very small $(2.7 \mathrm{~nm})$ and slightly larger $(8.5 \mathrm{~nm})$ sizes, which are shown in Figure $8 \mathrm{a}$, by purple and blue arrows, respectively. The sample consists of particles that appear to be of spherical morphology and of polycrystalline nature is revealed by the ring SAED pattern. Indexing the SAED pattern also shows that the phase of the sample is brookite, which is in good agreement with the XRD analysis where the relative fraction of brookite in the sample was estimated to be $70 \%$. The $d$-spacing values were measured and are denoted in Figure $8 \mathrm{c}$. In addition, the morphology of the crystals shown in the TEM images in Figures 7 and 8 corroborates the morphology obtained by the Rietveld analysis of the diffractograms (Supporting Information, Figure S1).

Figure 9 shows TEM images of sample A5 (14 day synthesis time). The morphology of the sample appears to be a mixture of the previous two samples. The sample consists of areas of elongated needle-shaped crystals (as in the 3 day sample) and areas of almost round particles (as in the 56 day sample). This
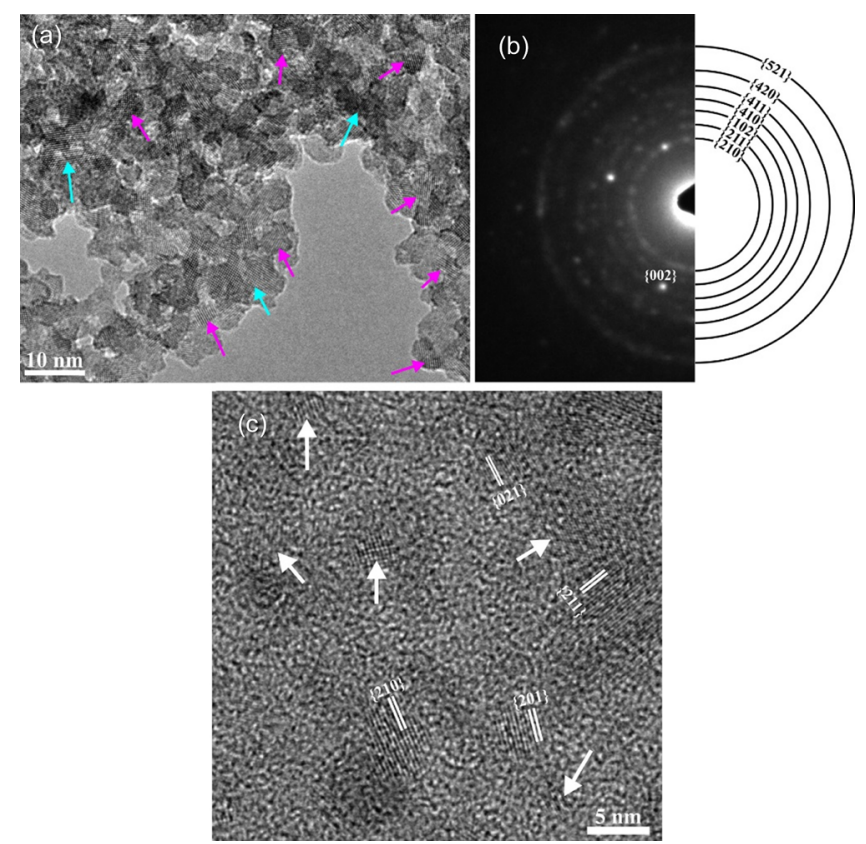

Figure 8. (a, c) HRTEM images and (b) SAED pattern of sample A9 (56 day synthesis time). Image (a) shows that the sample is polycrystalline, and purple arrows denote small crystals, whereas cyan arrows denote bigger crystals. The SAED ring pattern corresponds to the brookite phase. A high magnification of the sample is shown in (c) where the $d$-spacing values were measured and the brookite lattice planes are denoted.

is in agreement with the Rietveld analysis of the X-ray diffractograms where the samples were found to contain two main phases, rutile and brookite.

The TEM characterization is in good agreement with the XRD analysis, supporting the observation that initially the rutile phase is formed and the other phases are formed when the instantaneous precursor concentration decreases. Moreover, our results show that the rutile phase is not converted to the anatase and brookite phases, but that they form independently of the rutile phase. This is supported by the observation that the yield of the rutile phase does not decrease with synthesis time, as brookite and anatase are formed. A more probable explanation is that other chemical pathways become more feasible as the chemical environment changes as the reaction proceeds, leading to formation of the more thermodynamically stable anatase and brookite nanoparticle phases.

\section{CONCLUSIONS}

A low-temperature low-pH synthesis of titania was studied, and the effect of precursor concentration on polymorph selectivity was evaluated. During the reaction, rutile is initially formed, whereas brookite and anatase are preferentially formed at later stages. It was confirmed that a solid-solid transformation of rutile to anatase or brookite phases is improbable under reaction conditions, that anatase and brookite formations occur instead from unreacted precursors in the solution, and that the time dependent polymorph selectivity is instead related to changes in the chemical environment of the system. At high titania precursor concentration, mainly rutile was formed, but as the concentration decreases, brookite and anatase are favored. 

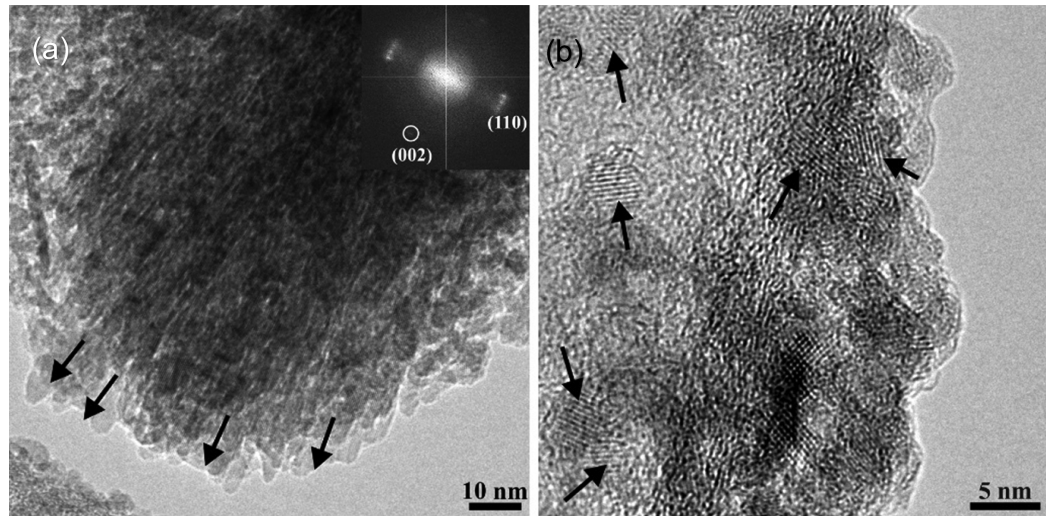

Figure 9. Bright field TEM images of sample A5, 14 day synthesis time (a) in low magnification, the inset is the FFT corresponding to the central part of the image, and (b) in high resolution. The sample shows mixture of randomly oriented spherical crystals and needle-shape crystals. In (a), the arrows denote the direction of the needle-shaped crystals and in (b), the arrows denote some of the spherical-shaped crystals.

\section{EXPERIMENTAL METHODS}

Reagent mixtures were prepared using hydrochloric acid $(37 \%$ Sigma-Aldrich), ethanol (99.5\% Solveco), and titanium $n$ butoxide (97\% Sigma-Aldrich). Three series of samples, with varying concentrations of titanium $n$-butoxide, were prepared. In series $\mathrm{A}, 1.5 \mathrm{~g}$ of $5 \mathrm{M} \mathrm{HCl}, 3 \mathrm{~g}$ of ethanol, and $1.5 \mathrm{~g}$ of titanium $n$-butoxide were added to a $20 \mathrm{~mL}$ glass vial. The glass vial was capped, and the content shaken until the mixture became clear after which the vial was placed in an oven at 40 ${ }^{\circ} \mathrm{C}$. After the chosen synthesis time, the sample mixture was removed, and the formed titania particles were retrieved and washed three times by ultracentrifugation using Beckman Coulter Ultracentrifuge Optima 100XL with a Type 90 Ti fixed angle rotor at $25000 \mathrm{~g}$ for $1 \mathrm{~h}$. The particles were then dried at $40{ }^{\circ} \mathrm{C}$ in an oven. Each sample is composed of the combined products from 4 to 10 vials. Series $B$ and $C$ were prepared in similar ways, but in series B, $1.5 \mathrm{~g}$ of $5 \mathrm{M} \mathrm{HCl}, 3.6 \mathrm{~g}$ of ethanol, and $0.75 \mathrm{~g}$ of titanium $n$-butoxide were used, and in series $\mathrm{C}$, $1.5 \mathrm{~g}$ of $5 \mathrm{M} \mathrm{HCl}, 2.1 \mathrm{~g}$ of ethanol, and $2.6 \mathrm{~g}$ of titanium $n$ butoxide were used.

Powder X-ray diffraction (PXRD) measurements were conducted on a Rigaku SmartLab diffractometer with $\mathrm{Cu} \mathrm{K} \alpha$ source (1.54056 $\AA$ ) using the Bragg-Brentano optics. The Rietveld refinements were performed using Fullprof software. ${ }^{21}$ The background was described by a four-coefficient Chebyshev polynomial function. The site occupancies, positions, and atomic displacement parameters were fixed to literature values. $^{22,23}$ The peak profiles were described using the Thompson-Cox-Hastings pseudo-Voight function. To model the anisotropic size broadening, a linear combination of maximum four spherical harmonic functions was used. A $\mathrm{LaB}_{6}$ NIST standard, measured at the same conditions, was modeled to obtain the instrumental broadening. Quantitative XRD measurements were performed on all samples, by adding a small known amount of diamond to the sample as an internal standard.

For structural characterization, selected area electron diffraction (SAED), and high-resolution transmission electron microscopy (HRTEM) were employed. The samples were crushed in a mortar, dispersed in ethanol, dropped onto a carbon-coated cupper grid, and let dry at room temperature. The experiments were performed on a Tecnai G2 microscope, operating at $200 \mathrm{kV}$.

\section{ASSOCIATED CONTENT}

Supporting Information

The Supporting Information is available free of charge on the ACS Publications website at DOI: 10.1021/acsomega.8b03440.

Lattice parameters, refinement data, XRD diffractograms and crystal morphology, and polymorph selectivity (PDF)

\section{AUTHOR INFORMATION}

\section{Corresponding Author}

*E-mail: anders.palmqvist@chalmers.se.

ORCID ${ }^{\circ}$

Gunnar Símonarson: 0000-0002-6744-3493

Bo B. Iversen: 0000-0002-4632-1024

Anders E.C. Palmqvist: 0000-0002-7579-3936

Notes

The authors declare no competing financial interest.

\section{ACKNOWLEDGMENTS}

This work has been carried out with support from the Swedish Energy Agency, project number 38340-1. G.S. thanks the Landsvirkjun Energy Research Fund for financial support. The Danish National Research Foundation is thanked for support (DNRF93).

\section{REFERENCES}

(1) Fujishima, A.; Honda, K. Electrochemical Photolysis of Water at a Semiconductor Electrode. Nature 1972, 238, 37-38.

(2) O’Regan, B.; Grätzel, M. A Low-Cost, High-Efficiency Solar Cell Based on Dye-Sensitized Colloidal $\mathrm{TiO}_{2}$ Films. Nature 1991, 353, 737-740.

(3) Lam, S. W.; Soetanto, A.; Amal, R. Self-Cleaning Performance of Polycarbonate Surfaces Coated with Titania Nanoparticles. J. Nanopart. Res. 2009, 11, 1971-1979.

(4) Karlsson, J.; Jimbo, R.; Fathali, H. M.; Schwartz-Filho, H. O.; Hayashi, M.; Halvarsson, M.; Wennerberg, A.; Andersson, M. In Vivo Biomechanical Stability of Osseointegrating Mesoporous $\mathrm{TiO}_{2}$ Implants. Acta Biomater. 2012, 8, 4438-4446.

(5) Sakatani, Y.; Grosso, D.; Nicole, L.; Boissière, C.; de A. A. SolerIllia, G. J.; Sanchez, C. Optimised Photocatalytic Activity of Grid-like Mesoporous $\mathrm{TiO}_{2}$ Films : Effect of Crystallinity, Pore Size Distribution, and Pore Accessibility. J. Mater. Chem. 2006, 16, 77-82. 
(6) Li, Y.; White, T. J.; Lim, S. H. Low-Temperature Synthesis and Microstructural Control of Titania Nano-Particles. J. Solid State Chem. 2004, 177, 1372-1381.

(7) Sugimoto, T.; Zhou, X. Synthesis of Uniform Anatase $\mathrm{TiO}_{2}$ Nanoparticles by the Gel-Sol Method. J. Colloid Interface Sci. 2002, $252,347-353$.

(8) Andersson, M.; Österlund, L.; Ljungström, S.; Palmqvist, A. Preparation of Nanosize Anatase and Rutile $\mathrm{TiO}_{2}$ by Hydrothermal Treatment of Microemulsions and Their Activity for Photocatalytic Wet Oxidation of Phenol. J. Phys. Chem. B 2002, 106, 10674-10679.

(9) Yang, J.; Mei, S.; Ferreira, J. M. F. Hydrothermal Synthesis of Nanosized Titania Powders: Influence of Peptization and Peptizing Agents on the Crystalline Phases and Phase Transitions. J. Am. Ceram. Soc. 2000, 83, 1361-1368.

(10) Mi, J.-L.; Jensen, K. M. OØ.; Tyrsted, C.; Bremholm, M.; Iversen, B. B. In Situ Total X-Ray Scattering Study of the Formation Mechanism and Structural Defects in Anatase $\mathrm{TiO}_{2}$ Nanoparticles under Hydrothermal Conditions. CrystEngComm 2015, 17, 68686877.

(11) Nilsson, E.; Furusho, H.; Terasaki, O.; Palmqvist, A. E. C. Synthesis of Nanoparticulate Anatase and Rutile Crystallites at Low Temperatures in the Pluronic F127 Microemulsion System. J. Mater. Res. 2011, 26, 288-295.

(12) Andersson, M.; Kiselev, A.; Österlund, L.; Palmqvist, A. E. C. Microemulsion-Mediated Room-Temperature Synthesis of HighSurface-Area Rutile and Its Photocatalytic Performance. J. Phys. Chem. C 2007, 111, 6789-6797.

(13) Nilsson, E.; Sakamoto, Y.; Palmqvist, A. E. C. LowTemperature Synthesis and HRTEM Analysis of Ordered Mesoporous Anatase with Tunable Crystallite Size and Pore Shape. Chem. Mater. 2011, 23, 2781-2785.

(14) Elgh, B.; Palmqvist, A. E. C. Controlling Anatase and Rutile Polymorph Selectivity during Low-Temperature Synthesis of Mesoporous $\mathrm{TiO}_{2}$ Films. J. Mater. Chem. A 2014, 2, 3024-3030.

(15) Elgh, B.; Palmqvist, A. E. C. A Facile Low-Temperature Synthesis of $\mathrm{TiO}_{2}$ Nanoparticles with Excellent Polymorph Control. J. Sol-Gel Sci. Technol. 2015, 76, 395-401.

(16) Zhang, H.; Banfield, J. F. Thermodynamic Analysis of Phase Stability of Nanocrystalline Titania. J. Mater. Chem. 1998, 8, 20732076.

(17) Yu, J.; Yu, J. C.; Leung, M. K.-P.; Ho, W.; Cheng, B.; Zhao, X.; Zhao, J. Effects of Acidic and Basic Hydrolysis Catalysts on the Photocatalytic Activity and Microstructures of Bimodal Mesoporous Titania. J. Catal. 2003, 217, 69-78.

(18) Yu, J. G.; Yu, J. C.; Cheng, B.; Hark, S. K.; Iu, K. The Effect of $\mathrm{F}^{-}$-Doping and Temperature on the Structural and Textural Evolution of Mesoporous $\mathrm{TiO}_{2}$ Powders. J. Solid State Chem. 2003, 174, 372380.

(19) Gribb, A. A.; Banfield, J. F. Particle Size Effects on Transformation Kinetics and Phase Stability in Nanocrystalline $\mathrm{TiO}_{2}$. Am. Mineral. 1997, 82, 717-728.

(20) Zhang, H.; Banfield, J. F. Understanding Polymorphic Phase Transformation Behavior during Growth of Nanocrystalline Aggregates: Insights from $\mathrm{TiO}_{2}$. J. Phys. Chem. B 2000, 104, 3481-3487.

(21) Rodríguez-Carvajal, J. Recent Advances in Magnetic Structure Determination by Neutron Powder Diffraction. Phys. B Condens. Matter 1993, 192, 55-69.

(22) Baur, W. H. Über Die Verfeinerung Der Kristallstrukturbestimmung Einiger Vertreter Des Rutiltyps: $\mathrm{TiO}_{2}, \mathrm{SnO}_{2}, \mathrm{GeO}_{2}$ Und $\mathrm{MgF}_{2}$. Acta Crystallogr. 1956, 9, 515-520.

(23) Djerdj, I.; Tonejc, A. M. Structural Investigations of Nanocrystalline $\mathrm{TiO}_{2}$ Samples. J. Alloys Compd. 2006, 413, 159-174.

(24) Hanaor, D. A. H.; Sorrell, C. C. Review of the Anatase to Rutile Phase Transformation. J. Mater. Sci. 2011, 46, 855-874.

(25) Birgisson, S.; Saha, D.; Iversen, B. B. Formation Mechanisms of Nanocrystalline $\mathrm{MnO}_{2}$ Polymorphs under Hydrothermal Conditions. Cryst. Growth Des. 2018, 18, 827-838.
(26) Zhang, H.; Chen, B.; Banfield, J. F.; Waychunas, G. A. Atomic Structure of Nanometer-Sized Amorphous $\mathrm{TiO}_{2}$. Phys. Rev. B Condens. Matter Mater. Phys. 2008, 78, 1-12.

(27) Mamakhel, A.; Tyrsted, C.; Boøjesen, E. D.; Hald, P.; Iversen, B. B. Direct Formation of Crystalline Phase Pure Rutile $\mathrm{TiO}_{2}$ Nanostructures by a Facile Hydrothermal Method. Cryst. Growth Des. 2013, 13, 4730-4734.

(28) Brinker, C. J.; Scherer, G. W. Sol-Gel Science; Academic Press: London, 1990.

(29) Livage, J.; Henry, M.; Sanchez, C. Sol-Gel Chemistry of Transition Metal Oxides. Prog. Solid State Chem. 1988, 18, 259-341. 